VALQUÍRIA ROVERAN ${ }^{1}$

Maria Antonieta Longo Galvão Silva ${ }^{2}$

LUCIA YAMANO ${ }^{1}$

LIIIAN PaIVA RODRIGUes ${ }^{2}$

MÔNICA LOPEZ VASQUEZ ${ }^{2}$

SEBASTIÃO PIATO ${ }^{3}$

Artigo original

Palavras-chave

Ruptura prematura de membranas fetais

Citocinas

Fator de necrose tumoral alfa

Sistema imune

Gravidez/imunologia

Corioamnionite/diagnóstico

Keywords

Fetal membranes, premature rupture

Cytokines

Tumor necrosis factor-alpha

Immune system

Pregnancy/immunology

Chorioamnionitis/diagnosis

\section{Expressão local do fator de necrose tumoral alfa na ruptura prematura de membranas}

\author{
Local expression of tumor necrosis factor-alpha on premature \\ rupture of membranes
}

\section{Resumo}

OBJETIVO: comparar a expressão do fator de necrose tumoral alfa (TNF- $\alpha$ ) em membranas ovulares com ruptura prematura (RPM) e com ruptura oportuna das mesmas; verificar a associação entre a expressão do TNF- $\alpha$ em membranas ovulares e o grau de corioamnionite das mesmas e correlacionar a expressão do TNF- $\alpha$ e o tempo de ruptura das membranas. MÉTODOS: foram analisadas as membranas ovulares de 31 parturientes com RPM, com idade gestacional acima de 34 semanas, e de 14 parturientes com ruptura oportuna das membranas, com idade gestacional igual ou maior de 37 semanas. A detecção da corioamnionite foi feita por meio de estudo histopatológico. A avaliação da expressão do TNF- $\alpha$ foi feita por meio de técnica imunoistoquímica, na qual foi empregado o método streptavidina-biotina-peroxidase (LSAB). RESULTADOS: o tempo médio de ruptura foi de 16,6 horas. A frequência da expressão de TNF- $\alpha$, nos Grupos Controle e Estudo, não mostrou diferença significante $\left(\chi^{2}=6,6 ; p=0,08\right)$. No Grupo Estudo, houve correlação entre o grau de corioamnionite e a intensidade da expressão de TNF- $\alpha$ (coeficiente de Spearman (Rs)=0,4; $p=0,02)$. CONCLUSÕES: não houve diferença significante entre as expressões do TNF- $\alpha$ em membranas ovulares com ruptura prematura e com ruptura oportuna das mesmas; no Grupo Estudo, constatou-se associação significante entre a expressão do TNF- $\alpha$ e o grau de corioamnionite e não houve associação entre o tempo de ruptura e a intensidade da expressão do TNF- $\alpha$

\section{Abstract}

PURPOSE: to compare the expression of tumor necrosis factor-alpha (TNF- $\alpha$ ) in ovular membranes with premature rupture (MPR) and with opportune rupture; to verify the association between the expression of the TNF- $\alpha$ in ovular membranes and the degree of chorioamnionitis, correlating the expression of the TNF- $\alpha$ and the membranes' time of rupture. METHODS: ovular membranes from 31 parturients with MPR, with gestational ages over 34 weeks, and from parturients with opportune membranes' rupture, with gestational ages equal or over 37 weeks. Chorioamnionitis detection has been done by histopathological analysis. The evaluation of the TNF- $\alpha$ expression has been done by immune-histochemical technique, using the labile streptavidin-biotin-peroxidase (LSAB) method. RESULTS: the average rupture time was 16.6 hours. The ratio of the TNF- $\alpha$ expression in the Control and Study Groups did not show a significant difference $\left(\chi^{2}=6.6 ; p=0.08\right)$. In the Study Group, there was no correlation between the degree of chorioamnionitis and the intensity of TNF- $\alpha$ expression (Spearman's coefficient $(R s)=0.4 ; p=0.02$ ). CONCLUSIONS: there was no significant difference between the TNF- $\alpha$ expression in ovular membranes with premature or opportune rupture; in the Study Group, there was significant association between TNF- $\alpha$ expression and the degree of chorioamnionitis, and there was no association between rupture time and the intensity of TNF- $\alpha$ expression.
Correspondência:

Valquíria Roveron

Rua Dr Cesário Mota Jr, 112 - Vila Buarque (EP: 01221010 - São Paulo (SP), Brasi Fone/fax: 11 2176-7384 E-mail: valrover@terra.com.br ou doutoraroveran@terra.com.br
Departamento de Obstetrícia e Ginecologia da Irmandade da Santa Casa de Misericórdia de São Paulo - ISCMSP - São Paulo (SP), Brasil.

'Médica Assistente do Departamento de Obstetrícia e Ginecologia da Irmandade da Santa Casa de Misericórdia de São Paulo ISCMSP - São Paulo (SP), Brasil; Professora Instrutora da Faculdade de Ciências Médicas da Santa Casa de São Paulo - São Paulo (SP), Brasil.

${ }^{2}$ Médica Assistente do Departamento de Ciências Patológicas da Irmandade da Santa Casa de Misericórdia de São Paulo - ISCMSP - São Paulo (SP), Brasil; Professora-assistente da Faculdade de Ciências Médicas da Santa Casa de São Paulo - São Paulo (SP), Brasil.

${ }_{3}^{3}$ Professor Titular do Departamento de Obstetrícia e Ginecologia da Irmandade da Santa Casa de Misericórdia de São Paulo da Faculdade de Ciências Médicas da Santa Casa de São Paulo - São Paulo (SP), Brasil. 


\section{Introdução}

A ruptura prematura de membranas (RPM) é complicação obstétrica que expõe a gestante e o concepto a riscos elevados. Dentre as complicações relacionadas à RPM, cumpre ressaltar o aparecimento de quadros infecciosos ${ }^{1,2}$.

Apesar das investigações realizadas até o presente, ainda permanecem dúvidas se a RPM é responsável pelo desenvolvimento de infecção ovular ou se este evento constitui-se na causa da ruptura das membranas.

Do mesmo modo que ocorre nos demais processos infecciosos, a suscetibilidade ao aparecimento da infecção das membranas relaciona-se com integridade da resposta imune do organismo materno ${ }^{3,4}$.

Consideradas de grande importância na regulação da resposta imune, as citocinas são polipeptídios habitualmente ausentes no plasma em condições estéreis. Dentre as mesmas, o fator de necrose tumoral alfa (TNF- $\alpha$ ) desempenha relevante papel nos processos infecciosos ${ }^{5,6}$.

A principal função do TNF- $\alpha$ é promover a resposta imune, por meio do recrutamento de neutrófilos e monócitos para o local da infecção, além de ativá-los. Constitui-se no principal mediador da resposta inflamatória aguda contra bactérias Gram-negativas e outros microorganismos ${ }^{5}$. Existem poucos trabalhos sobre expressão de TNF- $\alpha$ em membranas com ruptura prematura, sendo que as primeiras investigações surgiram na década de $1990^{7,8}$. Alguns estudos mais recentes abordaram o tema em questão, porém, a pesquisa de TNF- $\alpha$ foi realizada em placenta, líquido amniótico, sangue periférico e de cordão umbilical, mas não houve trabalho com utilização de membranas amnióticas ${ }^{9-15}$.

Acredita-se na importância da busca por esclarecimentos sobre a fisiopatologia da RPM, principalmente no que se refere à associação desta complicação obstétrica à presença de processos infecciosos. Assim, face à escassez de trabalhos relacionados ao tema e ao provável envolvimento do TNF- $\alpha$ na presença de infecção em casos de RPM, justifica-se a realização do presente estudo, que teve como objetivos comparar a expressão do TNF- $\alpha$ em membranas ovulares com ruptura prematura e com ruptura oportuna das mesmas; verificar a associação entre a expressão do TNF- $\alpha$ em membranas ovulares e o grau de corioamnionite das mesmas, além de correlacionar a expressão do TNF- $\alpha$ e o tempo de ruptura das membranas.

\section{Métodos}

Este estudo obteve aprovação do Comitê de Ética em Pesquisa da Irmandade da Santa Casa de Misericórdia de São Paulo (ISCMSP).
O material do presente estudo constou de membranas ovulares provenientes dos partos de 45 mulheres, que ocorreram no complexo hospitalar da ISCMSP, no período de Julho a Dezembro de 2006. Os casos foram divididos em Grupo Estudo, no qual foram incluídas 31 parturientes que apresentaram ruptura prematura das membranas ovulares, e em Grupo Controle, formado por 14 parturientes com ruptura oportuna das membranas.

Em relação às 31 pacientes do Grupo Estudo, a idade gestacional encontrava-se acima de 34 semanas na ocasião da ruptura das membranas. As 14 pacientes que constituíram o Grupo Controle apresentavam idade gestacional igual ou maior de 37 semanas.

Os critérios de exclusão adotados para a formação de ambos os grupos foram presença de sinais clínicos de infecção (temperatura axilar $>37,8^{\circ} \mathrm{C}$, taquicardia, fisometria e hipersensibilidade uterina); doenças clínicas de repercussão sistêmica; outras doenças obstétricas - excetuando-se RPM no Grupo Estudo. Para o Grupo Controle, o achado histopatológico de corioamnionite foi considerado critério de exclusão, o que ocorreu em 6 dos 20 casos iniciais, restando, portanto 14 casos incluídos neste grupo.

A população estudada apresentou, em média, idade de 23,3 anos no Grupo Controle e 24,2 anos no Grupo Estudo, idade gestacional de 39,6 semanas no Grupo Controle e 38 semanas no Grupo Estudo e ambos os grupos foram constituídos, em sua maioria, por primíparas.

Para o exame histopatológico, as membranas ovulares de ambos os grupos, ainda inseridas nas placentas, foram recolhidas no pós-parto imediato, armazenadas em solução de formaldeído a $10 \%$, e encaminhadas para o exame das margens correspondentes ao sítio da ruptura. Para a realização do exame histopatológico das membranas ovulares, procedeu-se, inicialmente, à sua separação da placenta. Em seguida, foi cortada uma tira de membrana medindo aproximadamente $3 \mathrm{~cm}$ de largura, que foi enrolada com o sítio de ruptura no centro, constituindo o chamado jelly roll, para posterior corte. Os espécimes foram desidratados em álcool etílico, clareados pelo xilol e embebidos em parafina, para confecção dos blocos. Para o preparo das lâminas, os blocos foram cortados por meio de micrótomo calibrado para espessura de 4 $\mu \mathrm{m}$. Os cortes histológicos obtidos foram corados pelo método de hematoxilina-eosina (HE) e a leitura foi feita em microscópio óptico comum.

Por meio deste exame histopatológico, procurou-se estabelecer a presença ou ausência de corioamnionite. Esta foi definida histologicamente pela presença de células inflamatórias, especialmente leucócitos polimorfonucleares (PMN), nas membranas amnióticas ${ }^{16,17}$. Os achados histológicos foram graduados de 0 a 3 , sendo classificados de acordo com a densidade do infiltrado leucocitário, 
determinada pela contagem de PMN em: (0) ausência de corioamnionite, ou seja, menos de 5 PMN por campo; (1) corioamnionite leve, com 5 a $10 \mathrm{PMN}$ por campo; (2) corioamnionite moderada, apresentando de 10 a 20 PMN por campo e (3) corioamnionite acentuada, ou seja, presença difusa de PMN.

A técnica de imunoistoquímica para avaliação da expressão do TNF- $\alpha$ nas membranas ovulares previamente blocadas dos Grupos Estudo e Controle consistiu no método streptavidina-biotina-peroxidase lábil (LSAB) ${ }^{18}$. Foram utilizados anticorpo primário anti-TNF- $\alpha$ (código AS210NA, RD System ${ }^{\circledR}$, USA) e kit de visualização contendo anticorpo secundário conjugado à peroxidase $\mathrm{e}$ cromógeno (Envision ${ }^{\circledR}$, DAKO, USA). O controle negativo foi_realizado omitindo-se o anticorpo primário; em seu lugar, foi adicionado soro não imune. Nesses casos, nenhuma marcação foi observada.

A positividade da reação para identificação do TNF- $\alpha$ foi marcada pela coloração marrom sépia, tomando-se como controle positivo da reação o padrão de coloração do TNF- $\alpha$ presente no citoplasma de macrófagos em cortes de amígdala humana. Quando presente, a positividade da reação marcou o citoplasma de células trofoblásticas da membrana amniótica com cor marrom sépia de aspecto granular. A intensidade da reação foi graduada de 0 a 3, consoante e porcentagem de células trofoblásticas coradas. Considerou-se grau 0 ou reação negativa, quando nenhuma célula apresentava cor marrom sépia; grau 1, quando havia coloração de menos de $50 \%$ das células; grau 2, quando havia reação positiva em 60 a 90\%; e grau 3, quando a reação era mais intensa, com coloração de praticamente todas as células trofoblásticas.

Para a análise estatística, o teste $t$ de Student foi utilizado para a comparação dos resultados nos Grupos Controle e Estudo quanto à variável "idade gestacional”, por confirmar a suposição de normalidade. $O$ teste não paramétrico de Mann-Whitney foi utilizado para comparação dos resultados dos Grupos Controle e Estudo quanto à idade e paridade.

Para verificar a existência de associação entre a expressão de TNF- $\alpha$ ao grau de corioamnionite, foi utilizado o coeficiente de correlação de Spearman. Utilizou-se o teste do $\chi^{2}$ para a comparação da expressão de TNF- $\alpha$ entre os Grupos Controle e Estudo. Para todos os testes, foi utilizado nível de significância de $5 \%(\mathrm{p}<0,05)$. Os softwares usados nas análises estatísticas foram o Statistical Package for Social Sciences 13.0 e o GraphPad InStat 3.06.

\section{Resultados}

Em relação à expressão de TNF- $\alpha$, o Grupo Controle apresentou reação negativa em $14,3 \%$ dos casos, $71,4 \%$ de reação positiva grau 1, 14,3\% de reação positiva grau 2 e
Tabela 1 - Frequência da expressão do TNF- $\alpha$ nos Grupos Controle e Estudo

\begin{tabular}{lcccc}
\hline Expressão de TNF- $\alpha$ & \multicolumn{2}{c}{ Controle n $\%$} & \multicolumn{2}{c}{ Estudo n \% } \\
\hline Total de casos & 14 & 100 & 31 & 100 \\
Grau 0 & 2 & 14,2 & 2 & 6,45 \\
Grau 1 & 10 & 71,4 & 12 & 38,7 \\
Grau 2 & 2 & 14,2 & 15 & 48,4 \\
Grau 3 & 0 & 0 & 2 & 6,4 \\
\hline
\end{tabular}

nenhum caso de reação grau 3. O Grupo Estudo apresentou reação negativa em $6,4 \%$ dos casos, $38,7 \%$ de reação positiva grau 1, 48,4\% de reação grau 2 e $6,4 \%$ de reação grau 3. Estes dados são apresentados na Tabela 1. A comparação da frequência da expressão de TNF- $\alpha$ nos Grupos Controle e Estudo pelo teste do $\chi^{2}$ não mostrou diferença significante entre os grupos $\left(\chi^{2}=6,6 ; \mathrm{p}=0,08\right)$.

A frequência de corioamnionite encontrada no Grupo Estudo foi: grau $0=9,7 \%$; grau $1=48,4 \%$; grau $2=35,5 \%$ e grau $3=6,5 \%$. Verificou-se, neste grupo, a existência de correlação significante entre o grau de corioamnionite e a intensidade da expressão de TNF- $\alpha$, por meio do cálculo do coeficiente de correlação de Spearman (coeficiente de Spearman $(\mathrm{Rs})=0,4 ; \mathrm{p}=0,02)$.

O tempo de ruptura médio foi de 16,6 horas. $\mathrm{O}$ coeficiente de correlação de Spearman não mostrou associação significante entre o tempo de ruptura das membranas e a intensidade da expressão de TNF- $\alpha(\mathrm{Rs}=0,34$; $\mathrm{p}=0,06)$.

\section{Discussão}

Dentre os diferentes aspectos controversos relacionados à RPM, ressaltam-se aqueles referentes ao aparecimento da corioamnionite e os problemas decorrentes da instalação dessa complicação. A existência de poucos estudos específicos relativos à expressão do TNF- $\alpha$ na ruptura prematura de membranas e as dúvidas referentes ao papel dos processos infecciosos encontrados nessa complicação obstétrica motivaram a realização do presente estudo.

O Grupo Estudo, apesar de não apresentar nenhuma evidência clínico-laboratorial de infecção, apresentou corioamnionite leve (frequência de 48,4\%) ou moderada $(35,5 \%)$ na maioria dos casos. Esse achado é concordante com o de alguns autores ${ }^{19}$, que verificaram resposta inflamatória nas membranas de parturientes com RPM, em comparação com aquelas de parturientes com ruptura oportuna das mesmas.

Esses resultados parecem trazer contribuição no que se refere à controvérsia sobre a presença da corioamnionite. Alguns autores admitem ser a mesma consequência da ascensão de micro-organismos patogênicos presentes no trato genital inferior, através do canal cervical, após a perda da barreira protetora das membranas ovulares s,20-22 $^{2}$ 
enquanto que outros têm opinião de que a infecção das membranas e da cavidade amniótica ocorra previamente à ruptura das membranas, constituindo, portanto, fator causador da RPM ${ }^{1,4,19}$.

O estudo em questão sugere que pode haver presença de processo inflamatório local previamente à ruptura das membranas, o que corrobora os trabalhos que apontam ser a RPM de causa principalmente infecciosa ${ }^{19,23}$.

A comparação da expressão do TNF- $\alpha$ nos dois grupos não mostrou diferença significante. Houve, porém, predominância da reação grau 1 no Grupo Controle, com frequência de $71,4 \%$, enquanto a maioria do Grupo Estudo apresentou reação graus 2 e 3, com frequências de 48,4 e $38,7 \%$, respectivamente. Esses dados sugerem associação entre a intensidade da expressão do TNF- $\alpha$ ao grau de corioamnionite. Tal fato foi confirmado pelo coeficiente de correlação de Spearman, calculado entre estas variáveis, que mostrou positividade ( $\mathrm{Rs}=0,4 ; \mathrm{p}=0,02)$.

Os estudos relacionados na literatura, embora não possam ser diretamente comparados ao nosso, por apresentarem metodologia diferente, mostraram, em concordância com o atual, associação entre corioamnionite e aumento dos níveis de TNF- $\alpha^{7,8,12,24-26}$. Essa correlação positiva era resultado esperado nesta pesquisa, pois o é conhecido o papel do TNF- $\alpha$ como principal mediador da resposta inflamatória aguda contra os agentes infecciosos.

Constituiu-se preocupação deste trabalho verificar a possível influência do tempo de ruptura das membranas em relação à expressão do TNF- $\alpha$. O tempo de ruptura médio foi de 16,6 horas. Valendo-se do coeficiente de correlação de Spearman, não foi possível demonstrar associação entre o tempo de ruptura e a intensidade da expressão do TNF- $\alpha(R s=0,34 ; p=0,061)$. Sabe-se que o risco de corioamnionite é elevado com o prolongamento do período de latência, conforme apontam alguns trabalhos ${ }^{10,13,24,27}$. Esperava-se, portanto, correlação positiva entre este marcador de inflamação e o tempo de ruptura. A ausência de correlação significativa encontrada no estudo em questão talvez possa ser explicada pelo tempo médio de ruptura das membranas ter sido relativamente curto no Grupo Estudo, enquanto outros trabalhos mostram aumento da corioamnionite acima de 24 horas de ruptura.

O presente estudo, apesar de não se prestar à aplicação clínica direta, por ter sido realizada análise pós-natal dos casos, demonstra que existe atuação do sistema imune na RPM, o que condiz com a presença provável de processos infecciosos existentes previamente à ruptura das membranas - conhecimento este que proporciona aplicação prática quanto à prevenção da RPM, como, por exemplo, o tratamento pré-natal das infecções genitais.

Além disso, apesar de não ter sido notada diferença significante entre as expressões do TNF- $\alpha$ em membranas ovulares com ruptura prematura e com ruptura oportuna das mesmas, é fato que a pesquisa de TNF- $\alpha$ diretamente nas membranas ovulares pode ser utilizada nos casos de RPM que cursam com complicações infecciosas maternas e perinatais, para melhor elucidação do real foco infeccioso nos quadros duvidosos.

\section{Referências}

1. Mercer BM. Preterm premature rupture of the membranes: current approaches to evaluation and management. Obstet Gynecol Clin North Am. 2005;32(3):411-28.

2. Medina TM, Hill DA. Preterm premature rupture of membranes: diagnosis and management. Am Fam Physician. 2006;73(4):659-64.

3. Minkoff H. Prematurity: infection as an etiologic factor. Obstet Gynecol. 1983;62(2):137-44.

4. Newton ER. Preterm labor, preterm premature rupture of membranes, and chorioamnionitis. Clin Perinatol. 2005;32(3):571-600.

5. Abbas AK, Lichtman AH, Pober JS. Cellular and molecular immunology. 5th ed. Philadelphia: WB Saunders; 2000.

6. Forte WN. Imunologia básica e aplicada. Porto Alegre: Artmed; 2004

7. Beltrán Montoya J, Meráz Cruz N, Vadillo Ortega F. Identificación de nexos moleculares entre infección y ruptura prematura de membranas. Ginecol Obstet Méx. 1997;65(5):191-3.
8. Baud O, Emilie D, Pelletier E, Lacaze-Masmonteil T, Zupan $\mathrm{V}$, Fernandez $\mathrm{H}$, et al. Amniotic fluid concentrations of interleukin-1 beta, interleukin-6 and TNF-alpha in chorioamnionitis before 32 weeks of gestation: histological associations and neonatal outcome. Br J Obstet Gynaecol. 1999;106(1): 72-7.

9. Lonergan M, Aponso D, Marvin KW, Helliwell RJ, Sato TA, Mitchell $M D$, et al. Tumor necrosis factor-related apoptosis-inducing ligand (TRAIL), TRAIL receptors, and the soluble receptor osteoprotegerin in human gestational membranes and amniotic fluid during pregnancy and labor at term and preterm. J Clin Endocrinol Metab. 2003;88(8):3835-44.

10. McElrath TF, Allred EN, Leviton A; Development Epidemiology Network Investigators. Prolonged latency after preterm premature rupture of membranes: an evaluation of histologic condition and intracranial ultrasonic abnormality in the neonate born at $<28$ weeks of gestation. Am J Obstet Gynecol. 2003;189(3): 794-8. 
11. El-Shazly S, Makhseed M, Azizieh F, Raghupathy R. Increased expression of pro-inflammatory cytokines in placentas of women undergoing spontaneous preterm delivery or premature rupture of membranes. Am J Reprod Immunol. 2004;52(1):45-52.

12. Winkler $M$. Role of cytokines and other inflammatory mediators. BJOG. 2003;1 10 Suppl 20:118-23.

13. Hansen-Pupp I, Harling S, Berg AC, Cilio C, Hellström-Westas L, Ley D. Circulating interferon-gamma and white matter brain damage in preterm infants. Pediatr Res. 2005;58(5):946-52.

14. Satar M, Turhan E, Yapicioglu H, Narli N, Ozgunen FT, Cetiner S. Cord blood cytokine levels in neonates born to mothers with prolonged premature rupture of membranes and its relationship with morbidity and mortality. Eur Cytokine Netw. 2008;19(1): 37-41.

15. Velemínský M Jr, Prádná J, Velemínský M Sr, Tosner J. Relationship of amniotic-type placenta inflammation to pPROM, PROM and risk of early onset neonatal sepsis. Neuro Endocrinol Lett. 2008;29(4):447-50.

16. Fox H. The placenta and infection. In: Redman CWG, Sargent IL, Starkey PM, editors. The human placenta: a guide for clinicians and scientists. Oxford: Blackwell Scientific; 1993. p. 313-33.

17. Baergen RN. Manual of Benirschke and Kauffmann's pathology of the human placenta. New York: Springer; 2005.

18. Alves VAF, Bacchi CE, Vassalo J. Manual de imuno-histoquímica. São Paulo: Sociedade Brasileira de Patologia; 1999.

19. Naeye RL, Peters EC. Causes and consequences of premature rupture of fetal membranes. Lancet. 1980;1(8161):192-4.
20. Garite TJ. Premature rupture of the membranes. In: Creasy RK, Resnik R, editors. Maternal-fetal medicine: principles and practice. 3rd ed. Philadelphia: WB Saunders; 1994. p. 625-38.

21. Rivera ZR, Caba BF, Smirnow SM, Aguilera TJ, Larrain HA. Fisiopatología de la rotura prematura de las membranas ovulares em embarazos de pretérmino. Rev Chil Obstet Ginecol. 2004;69(3): 249-55.

22. Torrens MCT, Maioli LC, Carvalho RL. Ruptura prematura de membranas. Acta Méd (Porto Alegre). 2005;26:680-9.

23. Romero R, Ghidini A, Bahado-Singh R. Premature rupture of the membranes. In: Reece EA, Hobbins JC, Mahoney M, Petrie RH, editors. Medicine of the fetus \& mother. Philadelphia: Lippincott; 1992. p. 1430-68.

24. Zhang W, Wang L, Zhao Y, Kang J. Changes in cytokine (IL-8, IL-6 and TNF-alpha) levels in the amniotic fluid and maternal serum in patients with premature rupture of the membranes. Zhonghua $\mathrm{Yi}_{\mathrm{i}}$ Xue Za Zhi (Taipei). 2000;63(4):31 1-5.

25. Shobokshi A, Shaarawy M. Maternal serum and amniotic fluid cytokines in patients with preterm premature rupture of membranes with and without intrauterine infection. Int J Gynaecol Obstet. 2002;79(3):209-15.

26. Døllner H, Vatten L, Halgunset J, Rahimipoor S, Austgulen R. Histologic chorioamnionitis and umbilical serum levels of pro-inflammatory cytokines and cytokine inhibitors. BJOG. 2002;109(5):534-9.

27. Maayan-Metzger A, Mazkereth R, Shani A, Kuint J. Risk factors for maternal intrapartum fever and short-term neonatal outcome. Fetal Pediatr Pathol. 2006;25(3):169-77. 